\title{
A risk forecasting process for nanostructured materials, and nanomanufacturing
}

\author{
Mark R. Wiesner*1,3 and Jean-Yves Bottero ${ }^{2,3}$
}

${ }^{1}$ Civil and Environmental Engineering, Duke University, Durham, North Carolina, USA

${ }^{2}$ CEREGE UMR 6635, CNRS; Université Paul Cézanne, Aix-en-Provence, France

${ }^{3}$ Center for the Environmental Implications of NanoTechnology (CEINT)/ International

Consortium for the Environmental Implications of NanoTechnology (iCEINT)

*corresponding author: wiesner@duke.edu

\section{Résumé}

Les nanomatériaux ont des propriétés nouvelles qui permettent de nouvelles applications depuis l'électronique moléculaire jusqu'à la production d'énergie. La prise en compte de leur impact potentiel sur la santé humaine et l'environnement nécessite des méthodes prédictives associées à leur emploi. Toutefois la très grande variété de ces nanomatériaux ne permet pas de traiter la question du risque au cas par cas. La prévision des risques, surtout pour un grand nombre de matériaux, est rendue compliquée par les incertitudes sur les quantités produites, les caractéristiques de ces matériaux et leur utilisation, les causes d'exposition, et le manque de données concernant leurs effets sur les organismes et les écosystèmes. Actuellement, une évaluation du risque associé à l'émergence des nanomatériaux manufacturés est donc impossible par des méthodes traditionnelles.

Une autre méthode, faisant appel à un processus évolutif semble plus appropriée pour analyser ces risques. Dans cet article, nous proposons qu'une telle méthode devrait inclure six ingrédients-clés: 1) la capacité à produire des prévisions associées à des niveaux d'incertitude pour des questions à court terme 2) la capacité à évaluer les sources pertinentes de nanomatériaux, 3) une approche systémique des impacts de l'utilisation et de la production des nanomatériaux prenant en compte le cycle de vie, au delà des approches toxicologiques, 4) la possibilité d'actualiser les prévisions des risques dès que des informations nouvelles sont connues, 5) un retour pour améliorer les connaissances, 6) la capacité à fournir un retour d'analyse pour diminuer l'impact des nanomatériaux via l'amélioration des procédés fabrication. Ce dernier point implique que le risque potentiel associé à un nanomatériau doit pouvoir être mis en relation avec ses propriétés, de telle sorte que telle ou telle de ses caractéristiques est un indicateur de risque. Ainsi le procédé d'évaluation des risques nécessite de s'intéresser à des questions à court-terme relatives à des nanomatériaux déjà dans le commerce mais aussi à des problèmes sur le long terme qui requièrent une recherché de base et des avancées théoriques. Dans l'article nous soulignerons et discuterons les besoins associés à chacun des six ingrédients-clés cités ci-dessus. 


\begin{abstract}
Nanomaterials exhibit novel properties that enable new applications ranging from molecular electronics to energy production. Proactive consideration of the potential impacts on human health and the environment resulting from nanomaterial production and use requires methods for forecasting risk associated with of these novel materials. However, the potential variety of nanomaterials is virtually infinite and a case-by-case analysis of the risks these materials may pose is not possible. The challenge of forecasting risk for a broad number of materials is further complicated by large degrees of uncertainty concerning production amounts, the characteristics and uses of these materials, exposure pathways, and a scarcity of data concerning the relationship between nanomaterial characteristics and their effects on organisms and ecosystems. A traditional risk assessment on nanomaterials is therefore not possible at this time. In its place, an evolving process is needed for analyzing the risks associated with emerging nanomaterials-related industries.

In this communication, we propose that such a process should include the following six key features: 1) the ability to generate forecasts and associated levels of uncertainty for questions of immediate concern, 2) a consideration of all pertinent sources of nanomaterials, 3) an inclusive consideration of the impacts of activities stemming from nanomaterial use and production that extends beyond the boundaries of toxicology and include full life cycle impacts, 4) the ability to adapt and update risk forecasts as new information becomes available, 5) feedback to improve information gathering and, 6) feedback to improve nanomaterial design. Feature \#6 implies that the potential risks of nanomaterials must ultimately be determined as a function of fundamental, quantifiable properties of nanomaterials, so that when these properties are observed in a new material, they can be recognized as indicators of risk. Thus, the required risk assessment process for nanomaterials addresses needs that span urgent, shortterm questions dealing with nanomaterials currently in commerce, to longer-term issues that will require basic research and advances in theory. In the following sections we outline issues surrounding each of these six features ad discuss.
\end{abstract}

Key Words: Risk assessment, Nanomaterials, Life Cycle, Bayesian Network

Mots-clés: Evaluation des risques, Nanomatériaux, Cycle de vie, réseau bayésien. 


\section{Immediate concerns vs. uncertainty in risk forecasts}

There are already hundreds of nano-based products currently on the market with an estimated market size that ranges from tens to hundreds of billions of dollars per year and is growing at double digits. Worldwide, there are thousands of nanotechnology-based patents per year and in 2006 roughly one quarter of them were in the area of nanomaterials [1].

The rapidly growing trend of incorporating nanomaterials into commercial products demands short-term decisions regarding the possible risks that these materials and uses may present. But what should be the criteria for prioritizing risk? A consideration of risk must address both hazard and exposure. We broadly define exposure here to refer to the total concentration of a given nanomaterial in a defined setting since the factors controlling bioavailability and uptake of nanomaterials are poorly understood. Indeed the unknowns surrounding nanomaterials include not only the nature of nanomaterials that may find their way into commerce, the properties of these materials, and even the uses and handling practices for nanomaterials, but the environmental transport, persistence, and bioactivities including toxicity. These high degrees of uncertainty strongly impact the reliability of risk forecasts with the goal of preemptively avoiding damages that might occur in an emerging industry. Thus, decision-making based on these forecasts must take into account not only the forecasted magnitude of risk, but the degree of uncertainty associated with that forecasts. In addition, risk forecasts must be placed in relationship to the forecasted benefits and uncertainty associated with these benefits.

One scenario for comparison of benefit and risk is shown in Figure 1 where the benefits of a new technology are initially overestimated and risk is under-estimated. Because of the specificity of need identified for a new technology, and the open-ended nature of anticipating risk, the uncertainty associated with estimating benefits may be smaller initially, while uncertainty surrounding risk may be large. In the case shown in Figure 1, additional information yields improved estimates of both benefit and risk such that as the system moves towards more perfect information, risk and benefit are indistinguishable within the bounds of the uncertainty.

Similar scenarios might be constructed where the risks clearly outweigh benefits and vise versa. In the case where benefits are comparable to or greater than risks, the possibility arises that early over estimates of risk due to uncertainty may stifle the development of what would ultimately be determined to be a beneficial technology. Timely production and updates of risk information are therefore critical to guiding nanotechnology development at early, sensitive stages in the trajectory of their development. 


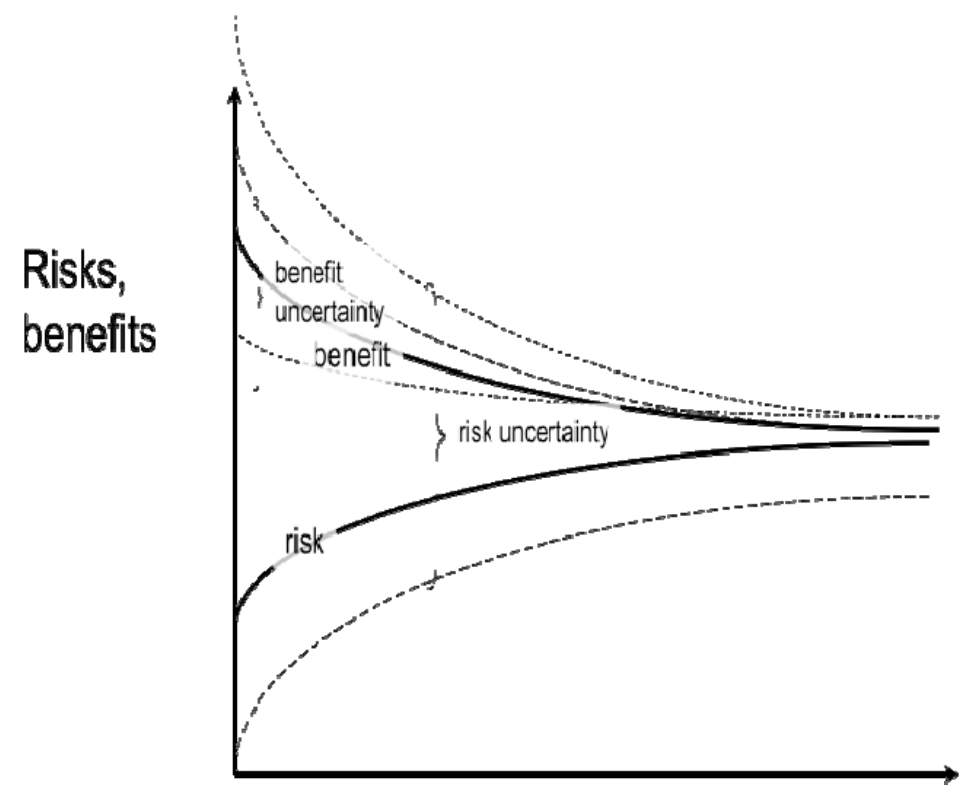

Time, information

Figure 1: Risks, benefits - time relationship

Work to date to identify the environmental, health, and safety (EHS) issues surrounding nanomaterials has been heavily weighted toward identifying possible nanomaterial hazards such as toxicity. Rapid screening for a given biological endpoint provides critical information on the potential hazard of a given nanomaterial. However, the ability to translate the results of these tests to actual human or ecosystem hazards has been limited by classic questions of doseresponse, appropriateness of animal models, ability to extrapolate from tests performed with cell lines, and the choice of endpoints (lethality, mutation, genotoxicity, developmental abnormalities, etc.). Moreover, the conditions of dosing in these tests typically do not account for environmental transformations of nanomaterials such as aggregation, adsorption, or dissolution. While these limitations, in addition to a lack of standardized protocols, make the generalization of negative results (i.e. no adverse effect) difficult at best, a positive result certainly signals the need for further scrutiny and caution. In addition, while reducing hazard through green chemistry and engineering approaches is not without merit, the properties of a material that produce the hazard may be closely related to those that make a given material useful in a specific application. An example is shown after in the case of $\mathrm{TiO}_{2}$. 
In contrast with work addressing hazard, there have been very few studies evaluating the factors controlling environmental exposure despite the fact the risk management strategies for nanomaterials, where needed, are likely to depend on exposure management. A risk management strategy rooted in a fundamental understanding of the possible pathways of exposure to nanomaterials leads to a broad array of options for managing risk, that spans protective devices for workers in nanomaterials fabrication industries, standards for product disposal or recycling, the use of pollution reduction equipment, changes in human behavior and, in extreme cases, an outright ban on the production of a given nanomaterial. An evaluation of exposure would therefore appear to be an excellent starting point in predicting the potential for risk posed by a given nanomaterial.

Indeed, in public testimony before a United States National Research Council committee in 2007 (see for example reference [2]) representatives from business, labor, environmental NGOs and consumer organizations all suggested that exposure should be the primary criteria for prioritizing EHS research on nanomaterials. However, considerable amounts of information are required to estimate environmental releases and exposure; information that is only partially available. Environmental releases are likely to depend on both the amounts produced and the number of products incorporating nanomaterials. Unfortunately, accurate estimates of amounts of nanomaterials known to be in production are difficult to obtain and may vary by several orders of magnitude depending on the source of information. Predictions of the production of future nanomaterials and markets are plagued by even greater degrees of uncertainty. In the absence of detailed information on nanomaterial markets and uses, an estimated "reservoir" of nanomaterial production regardless of their final use can be used to obtain first-order estimates of exposure that employ explicit, easily understood assumptions regarding the quantities of nanomaterials that enter the environment integrated over the entire life cycle of production through disposal [2]. Such an approach is consistent with the practice of regulating materials based on production volumes. Use of these estimates must be accompanied by probabilistic approaches to treating the wide ranges for estimated values with consideration given to the type of probability distribution assumed (e.g., uniform, log normal, etc.). Monte Carlo methods and Bayesian network methods can be used to formally represent uncertainty in models that link production estimates to environmental releases, transformations, and persistence.

\section{A consideration of all pertinent sources of nanomaterials}

While the production of engineered nanomaterials (ENMs) creates an immediate concern, a risk assessment for nanomaterials must take into account the relative magnitude of ENMs as sources compared with other sources of materials that may be identical or similar to 
ENMs. Nature produces a plethora of nano-scale particles in processes ranging from forest fires to bacterial metabolism. Human activities may also produce nano-scale particles by precipitation in waste streams, internal combustion engines, and other "incidental" sources. In some cases the materials produced are identical to ENMs as in the case of fullerenes produced in engineered, natural, or incidental combustion processes. Incidental carbon nanotubes (CNTs) and other fullerene-related nanocrystals have been reported to originate from propane stoves, wood fires, burning tires and other sources [3-5] and fullerene $\mathrm{C}_{60}$ has been found in geologic deposits [6], candle soot, and meteorites [7]. $\mathrm{TiO}_{2}$ nanoparticles, similar to ENMs, have been found downstream of hazard waste sites [8].

An assessment of exposure to nanomaterials must also address possible releases associated with various stages of fabrication, transport, processing and disposal; activities that make up what is referred to as the value chain of nanomaterial production and use. The nanomaterial value chain involves the production of basic building blocks of nanomaterials (often nanoparticles) and their incorporation in subsequent stages into products of increasing complexity. For example, engineered nanomaterials such as titanium dioxide might be modified with a tailored surface chemistry to yield suspensions that are then used to create various products ranging from thin films for self-cleaning windows to catalysts suspensions in water treatment. At each stage in the value chain, there exists the possibility of nanomaterial release and subsequent exposure to humans or ecosystems through the production, transport, use and disposal of nanomaterials and nanomaterial-containing products. Important factors to be identified in evaluating potential nanomaterial exposure at each stage in the value chain are the format that nanomaterials will be present in as commercial products, the potential for these materials to be released to the environment, and the transformations that those materials may undergo that may affect their subsequent potential for exposure. Indeed, due to modifications along the value chain or environmental transformations, the potential contact between humans and ecosystems outside of the work place will most likely involved nanomaterials there bear little resemblance to the initial material. 


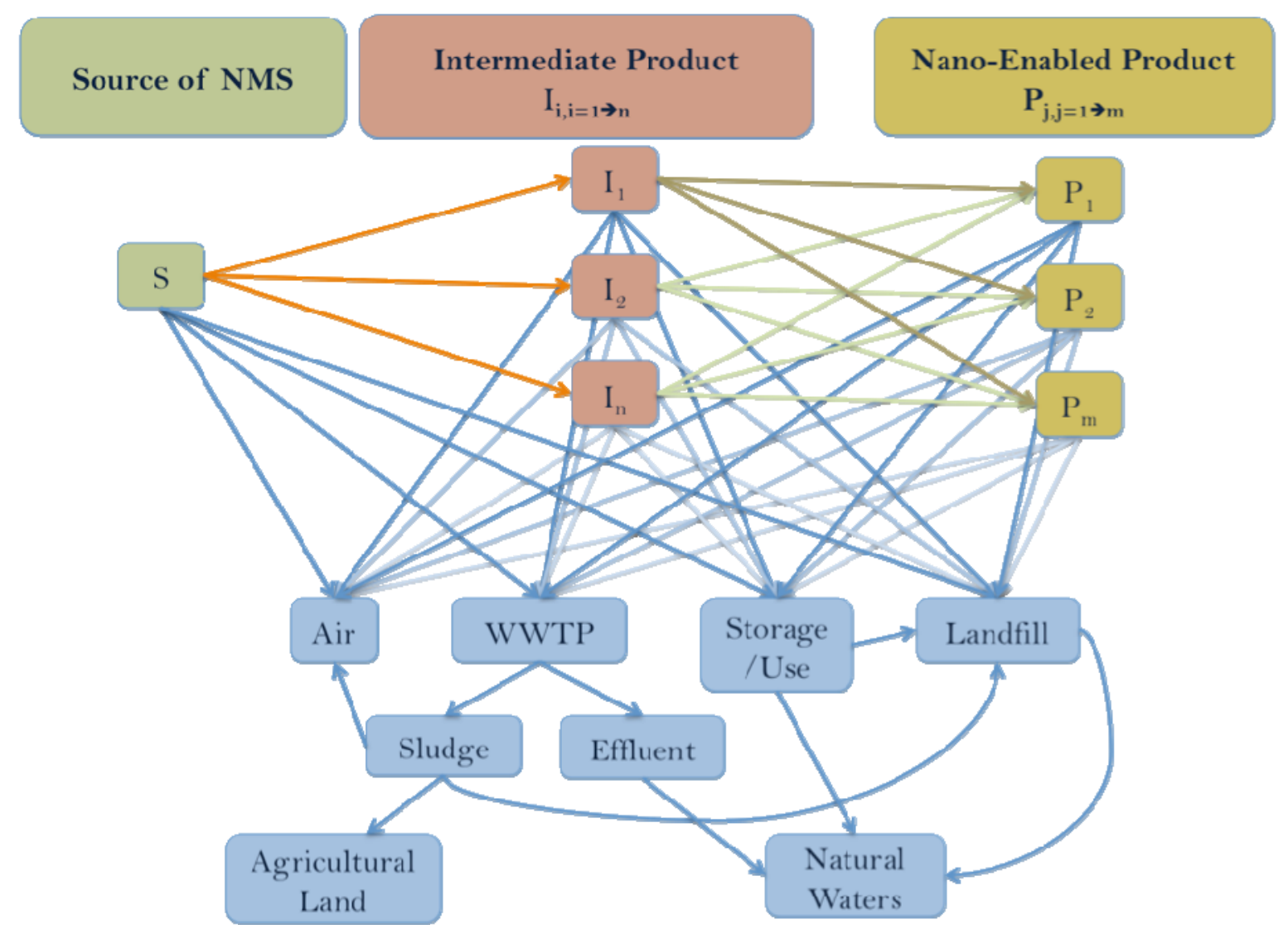

Figure 2: Simplified flow of nanomaterial through the production process and to the environment $(\mathrm{WWTP}=$ wastewater treatment plant $)$.

We formulate a framework for describing nanomaterial production and incorporation into products over various stages of a nanomaterial value chain where at each stage, there is the possibility of leakage into environmental compartments (Figure 2). Leakage can be aggregated over environmental compartments or over specific stages of the value chain. The flow of nanomaterials into various stages of the value chain and to environmental compartments can be generalized. We define the stage " $\mathrm{i}$ " of the value chain, where $\mathrm{i}=1$ corresponds to the nanomaterial source. At the source there is only one "product" (the raw nanomaterial) that is incorporated into any number of intermediate products (indexed as the " $\mathrm{j}_{\text {th" }}$ product) in stage 2 . Stage 2 products may then be incorporated into the nano-enabled products in stage 3 , etc. At an stage " $\mathrm{i}$ " the amount of nanomaterial represented in aggregate in product " $\mathrm{j}$ " is designated $\mathrm{P}_{\mathrm{i}, \mathrm{j}}$. Note that the number of products, i.e. the maximum value of $j$ depends on stage $i$. With this formalism, the amount of source product is $S=P_{1,1}$. 
The fraction of nanomaterials in products $\mathrm{P}_{\mathrm{i}, \mathrm{j}}$ that are incorporated into downstream products $P_{i+1, k}$ are designated as $f_{i, j, k}$. Similarly, there are "leakage" terms in to the environment, representing the fraction of product $\mathrm{P}_{\mathrm{i}, \mathrm{j}}$ that enters air, water, wastewater, etc. represented for example as $\mathrm{g}_{\mathrm{i}, \mathrm{j}, \mathrm{ww}}$ for the case of wastewater. Thus, the nanomaterials present in a product $\mathrm{k}$ at stage " $\mathrm{i}$ " of the value chain, $\mathrm{P}_{\mathrm{i}, \mathrm{k}}$ can be represented as function of materials flowing from the stages i-1 of the value chain:

$$
P_{i, k}=\sum_{j} P_{i-1, j} f_{i-1, j, k}
$$

For stage $\mathrm{i}=1, \mathrm{k}=1$ and $\mathrm{P}_{1,1}=\mathrm{S}$. At stage $\mathrm{i}=2$, the amount of nanomaterial present in product $\mathrm{k}$ comes only from the source $S$ and is given by:

$$
P_{2, k}=\sum_{j=1}^{1} S f_{1, j, k}=S f_{1,1, k}
$$

For stage $i=3$, nanomaterials in product $\mathrm{k}$ comes from all intermediate products present at stage $\mathrm{i}=2$ (indexed by j):

$$
P_{3, k}=\sum_{j} P_{2, j} f_{2, j, k}=S \sum_{j} f_{1,1, j} f_{2, j, k}
$$

The leakage/discharge to wastewater from stages 1, 2 and 3 is then given as:

$$
S g_{1,1, w w}+S \sum_{j} f_{1,1, j} g_{2, j, w w}+S \sum_{j} \sum_{l} f_{1,1, l} f_{2, l, j} g_{3, j, w w}=S * g_{w w}
$$

Conceptually, the description of all flows within this network, i.e. the $\mathrm{f}_{\mathrm{i}, \mathrm{j}, \mathrm{k}}$ coefficient between products and $\mathrm{g}_{\mathrm{i}, \mathrm{j}, \mathrm{ww}}$ between products and environment represents a very high demand for information on trends in commercialization, product use, product degradability, and nanomaterial transformation and transport. However aggregation across the value chain, or across receiving compartments such as wastewater, reduces the number of unknowns at the cost of loss of detail. For example, the amount of nanomaterial entering the wastewater compartment can be expressed as a product of the source of nanomaterials produced, $\mathrm{S}$, and the sum of products of coefficients representing all of the pertinent intermediate flows that yield a single constant, $\mathrm{g}_{\mathrm{ww}}$ in equation (4) that captures nanomaterial production and use profiles.

The value of $g_{w w}$ may not be known initially, but may be estimated from measurements of the quantities of nanomaterials in wastewater compared to nanomaterial production or estimated based on assumptions of use of these products.

Moreover, assumptions regarding the amount of nanomaterials entering wastewater are made explicit through the specification of a value for $\mathrm{g}_{\mathrm{ww}}$. This parameter can be used in a sensitivity 
analysis to explore "what-if" scenarios, may be calculated from actual measurements of nanomaterials in wastewater inflow if available, or may be estimated from fractions of other materials (e.g., PCBs) that may have been produced in the past.

Inclusive consideration of the impacts of activities stemming from nanomaterial use and production

Greater effort to estimate exposure does not imply that such work should be done at the exclusion of hazard or impacts assessment. To the contrary, there is a need for rapid screening of nanomaterial hazards to organisms, the elucidation of mechanisms producing these hazards as well as a broad consideration of possible hazards to the complex ecosystems these organisms inhabit. Consideration of ecosystem-level impacts has, until now, been largely absent from the assessment of nanomaterial hazards. Most work on evaluating nanomaterial hazards has been in the realm of the traditional toxicology-based approach to hazard assessment that seeks to describe effects on individual organisms or populations that can be traced to origins at the biomolecular, genetic, or cellular level. These effects may range from subtle changes in gene expression and perturbations in the function of endocrine systems, to morphologic changes in organism development and toxicity. Much work remains in this area. There is not yet a widely accepted suite of responses such as nano-particle DNA interactions, inflammation, or membrane disruption that can be used to reliably predict the effects of nanomaterials. Most nanotoxicological investigations have used pristine material and do not account for transformations that may occur in physiological or environmental systems. Moreover, the properties of nanomaterials that may affect their biouptake and biodistribution are largely unknown. A bottom-up approach that builds from the simple to the complex, may not adequately capture effects of nanomaterials at the level of ecosystems. For example, an evaluation of the toxicity of DDT to humans does not lead one to an understanding of the effects on reproductive functions of this molecule in predatory birds, nor does an analysis of the toxicity of $\mathrm{CO}_{2}$ lead to a prediction of global warming. While it is not possible to anticipate all hazards, information regarding the long-term impact of nanomaterials on ecosystem functions such as carbon or nitrogen cycling, or the possible shift in populations performing these functions correspond to the more complex impacts that might be considered for new materials entering ecosystems. 


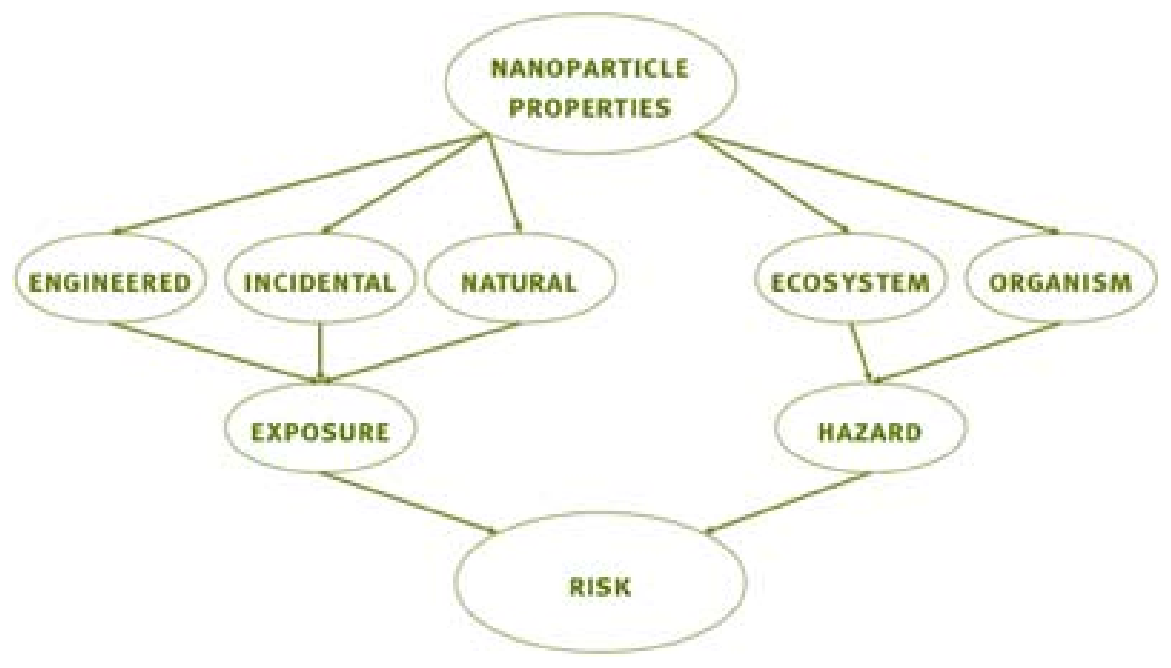

Figure 3: Evaluation of nanomaterial risks to ecosystems and organisms posed by multiple sources.

An additional set of hazards to be examined is the potential for "collateral damage" i.e. environmental impacts that arise from the production and use of nanomaterials rather than the nanomaterials themselves. In particular, the environmental impacts associated with upstream energy usage are likely to contribute significantly to the environmental footprint of nanomaterials production given the high energy inputs currently employed to create order at the nanoscale [9]. We speculate that these issues may greatly outweigh direct health or environmental impacts associated with an emerging nanomaterials industry. The first ever published work on nanomaterial risk assessment [10] dealt directly with issues of collateral damage. One of the important findings reported in this work was that methods for manufacturing nanomaterials tend to become "greener" with time; substituting, for example, less toxic solvents or implementing more energy-efficient procedures for fabricating nanomaterials. Subsequent work by others looking at carbon nanotube production [11] has shown that nanomaterial production may involve the production of non-nano wastes that pose significant hazards. 


\section{Adaption and updating of risk forecasts as new information becomes available}

The quality and nature of data concerning environment, health \& safety for nanoparticles is growing rapidly, and a risk forecasting process allow for improvements in forecasts over time. Short-term needs for screening of specific nanomaterials entering the market place are generating a growing data base of nanomaterial interactions. In addition to answering urgent questions regarding the safety of nanomaterials entering the market place, these data will contribute to longer-term efforts to map nanomaterial properties into the risks in the general case. However to make this step from the analysis of specific nanomaterials to the general case of evaluating a new hypothetical material, a robust risk prediction model is needed that can be easily updated with the growing knowledge base.

Such a model must relate the physical-chemical properties of ENMs to biological activity, ecosystem impacts, and the factors controlling exposure. These relationships are subtly more general than those referred to as quantitative structure activity relationships (QSARs) [12] in that second order nanomaterial properties of interest here may include novel properties such as redox reactivity or adsorptive capacity that derive from (and are therefore correlated with) structure parameters such as shape, electronic structure, crystal structure, surface defects, size, and surface functionality. However, the term QSAR is used here in the more general sense.

A QSAR for ENMs can be expressed mathematically as a mapping, $\mathrm{f}$, of ENMs properties to biological activity or response:

$$
B R=f\left(x_{1}, x_{2}, \mathrm{~K}\right)
$$

where BR is a biological response (e.g. the half maximal inhibitory concentration: IC50, the effective dose producing a therapeutic response in $50 \%$ of the people: $\mathrm{ED}_{50}$, the amount of a substance required to kill half a given population: $\left.\mathrm{LD}_{50}\right)$ and $x_{1}, x_{2}, \ldots$ are mathematical descriptors of ENM properties. In its simplest form, the mapping function $f$ may result from a multivariate regression. However, regression techniques are limited in that they are fundamentally interpolative relationships. Most applications require the use of more sophisticated nonlinear models for pattern recognition such as linear discriminant analysis, Knearest neighbor classifiers, neural networks, and support vector machines. Even these latter techniques are largely limited in their ability to extrapolate beyond the data sets used to produce or "train" the model.

An other model is the Bayesian probability network. It can be thought of as a graphical model with a series of nodes linked by arrows. The arrows indicate causal linkages among the nodes, and the nodes denote important system attributes. Each node is characterized by probabilities or probabilistic mathematical expressions that represent knowledge about these system attributes. The mathematical expressions may be 1) mechanistic descriptions such as 
chemical reaction kinetics, 2) empirical relationships such as linear regression models, or 3) relationships derived from expert judgment, depending on how much information we have about the relationships characterizing a particular node. The possible outcomes at each node are expressed probabilistically; thus a Bayes net $(\mathrm{BN})$ is a set of conditional probabilities describing a set of likely system responses with conditionality indicated by the arrows. The ability to incorporate mechanistic, empirical, and judgmental information makes the BN approach extremely flexible and facilitates an extension to non-traditional model endpoints of public concern.

In our case, BNs present four advantages:

- An enormous advantage of using Bayesian methods is this ability to work with uncertainty and the flexibility in data sources. Where information is lacking, relationships may simply take the form of "judgment" or heuristics. As more data become available, probability distributions may be constructed, and ultimately mechanistic models confirmed and integrated as submodels.

- A second important advantage is their ability to explicitly include mechanistic relationships that aid in predicting beyond the available data sets.

- Compared with neural nets, BNs offer better accuracy, are less sensitive to small data set size and are therefore more suited for frontier of knowledge that change rapidly and need frequent model reconstructions [13]. They can be updated with new knowledge in a manner that propagates this new information throughout the $\mathrm{BN}$, resulting in a revised and improved model.

- Because BNs characterize sets of conditional probabilities, a particular utility of Bayes nets is their ability to propagate uncertainty.

Creating a Bayesian Network begins with defining the variables that affect a system, and linking them together with one-way causal arrows to indicate which variables are the parents and which are children [14]. In this influence diagram, each variable has a finite set of mutually exclusive states; for example, the size of a nanoparticle might fall into a state of "less than $20 \mathrm{~nm}$ in diameter" or "more than $20 \mathrm{~nm}$ in diameter." After all the important variable nodes are documented, they can be simplified or combined into dimensionless variables based on factors such as which nodes are most influential and which nodes can be most readily measured or tracked. Causal relationships can be quantitatively defined in terms of their strength, yielding conditional probability relationships between parent variables with shared children. The probabilities propagate through the network, generating the resultant probability of a variable being in a particular state given the states of its parent and grandparent variables. Data or expert opinion can generate the initial probabilities, and as more data become available, these 
values are updated and refined or replaced with mechanistic relationships that are themselves functions of variables that are added to the network.

Causal relationships are not completely opaque to us. For example, we have been actively exploring the role of nanoparticle characteristics such as size, charge, and interactions with macromolecules in determining nanomaterial transport and transformation for over a decade [15-35]. In many cases the role of ionic strength and macromolecules in modifying the physical and chemical properties of nanoparticles has been well-established in the colloid science literature. Much is known about the role of London-van der Waals and electrical double-layer forces (the classic Derjaguin, Landau, Verwey and Overbeek or DLVO model, [36, 37]), steric interactions, hydration forces, and solvation forces as these phenomena affect particle surface chemistry. In many cases, mechanisms are understood and an "expert system" or statistical approach to describing such relationships is not needed.

However, it may not always be possible to simply apply the predictive relationships determined from colloid science or previous particle-toxicity studies directly to nanomaterials. For example, as particle size decreases to the nano range, electrostatic forces and steric interactions affecting particle stability may be important at scales that are larger than some dimensions of the nanoparticles. In these cases, the direct use of data or heuristics may be required to describe probabilities. As our understanding of the system increases over time, the system can be updated, replacing for example heuristics with new mechanism-based models.

Bayes nets are also particularly useful for adaptive implementation because of the natural way in which they can be updated as new information becomes available, via Bayes Theorem. Let us assume the value of a node is a vector $\theta$, and $\pi(\theta)$ is the probability of $\theta$. An additional information is then added: The value of a vector parameter $y$ influences the value of $\theta$ so that its probability should rather be defined as $\pi(\theta \mid y)$, i.e. the probability of $\theta$, after observing the new data, $y$, (the posterior probability of $\theta$ ). This new information can be introduced through a likelihood function $f(y \mid \theta)$ which incorporates the statistical relationships as well as the mechanistic or process relationships among the predictor and response variables. Bayes theorem gives the relationship between these $\pi(\theta), \pi(\theta \mid \mathrm{y})$ and $f(\mathrm{y} \mid \theta)$

$$
\pi(\theta / y)=\frac{\pi(\theta) f(y / \theta)}{\int_{\Theta} \pi(\theta) f(y / \theta) d \theta}
$$

A highly simplified example of a BN is shown below in Figure 4. Though this does not show every variable that affects aggregate size, it shows a subset that illustrates the flexibility in data 
collection and the interactive process between modeling and laboratory experimentation for the case of the fullerol nanoparticle (hydroxylated $\mathrm{C}_{60}$ ). Experiments are carried out to elucidate the relationship between macromolecules of naturally occurring organic matter (NOM), in our case, tannic acid and ionic strength ( $\mathrm{NaCl}$ concentration) and the resulting aggregate size of fullerol molecules. Because $\mathrm{pH}$ was held constant at 7.2, it is grayed out in this example, but is left in the network as an influential factor. Note that other factors describing the nanomaterial itself are excluded from this simplified diagram since the material is held "constant" for this example. The probability distribution of the aggregate size of fullerol is $\pi(\theta)$. The vector $y$ has two components:

- $\mathrm{y}_{1}=0$ or 1: Natural organic matter is collected as a binary variable with states of "yes" for the presence of a set concentration of NOM or "no" for its absence.

- $\mathrm{y}_{2}$ is the $\mathrm{NaCl}$ concentration which is varied in a range from $0 \mathrm{M}$ to $0.3 \mathrm{M}$; These values are separated into bins that represent ranges of values

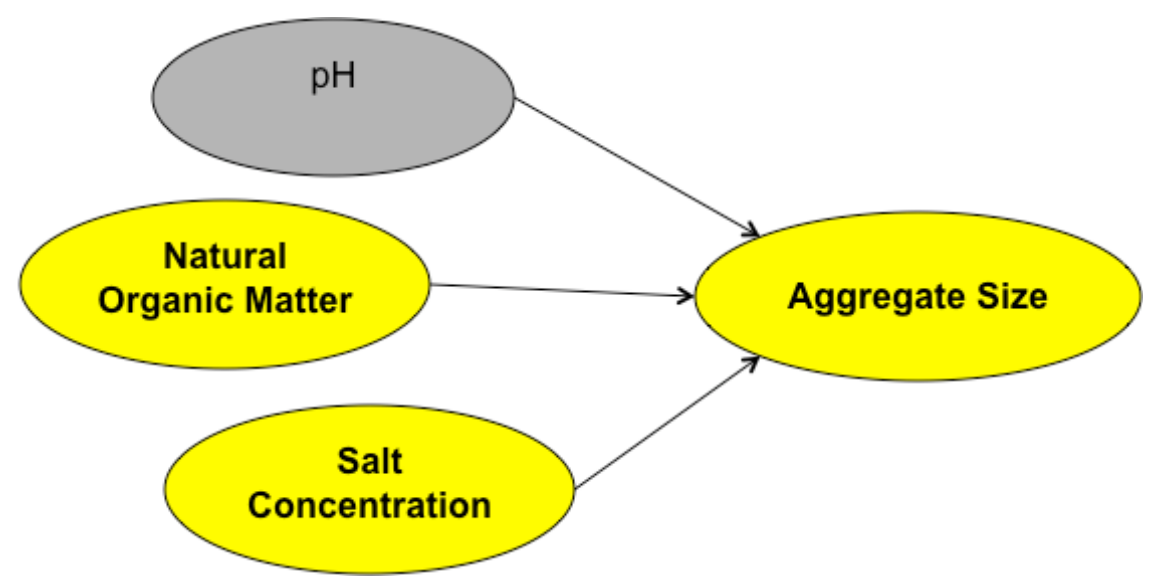

Figure 4: Bayes net structure of the fullerol aggregation system

The aggregate size was measured at a given time by dynamic light scattering and these values are also binned. The choices of how to bin each variable are important ones, because a balance must be struck between how much nuance is captured in the data (more detail with more bins) with how cumbersome the conditional probability tables are (more so with more bins). Relevant values and appropriate variable types are gathered from the experts that help develop the structure.

Our goal is to predict from a limited number of measurements the probability that, given the states of the parent variables, the child variable (size) is within a given state (size range). The conditional probability table generated from this data set, when binned with three ranges of salt concentration and four ranges of aggregate size values, illustrated in Table 2 below. 
Table 2: Conditional probability table for aggregate size (Example provided by C. Hendren and Eric Money, Duke University)

\begin{tabular}{|c|c|c|c|c|c|c|c|c|}
\hline \multirow{2}{*}{$\begin{array}{l}\text { For each box: } \\
\text { What is the probability } \\
\text { that the aggregate size } \\
\text { in this size bin for the } \\
\text { given set of conditions? }\end{array}$} & \multicolumn{2}{|c|}{$\begin{array}{c}\text { Size } \\
0<x<300\end{array}$} & \multicolumn{2}{|c|}{$\begin{array}{c}\text { Size } \\
300<x<600\end{array}$} & \multicolumn{2}{|c|}{$\begin{array}{c}\text { Size } \\
600<x<900\end{array}$} & \multicolumn{2}{|c|}{$\begin{array}{c}\text { Size } \\
900<x<1200\end{array}$} \\
\hline & \multicolumn{8}{|c|}{ Tannic acid } \\
\hline $\mathrm{NaCl}$ concentration & Yes & No & Yes & No & Yes & No & Yes & No \\
\hline $0.00-0.10$ & 0.875 & 0.3 & 0.125 & 0.6 & 0 & 0.08 & 0 & 0.025 \\
\hline $0.10-0.20$ & 1 & 0 & 0 & 0.25 & 0 & 0.4 & 0 & 0.35 \\
\hline $0.20-0.40$ & 0.3 & 0 & 0.7 & 0 & 0 & 0 & 0 & 1 \\
\hline
\end{tabular}

Looking at the upper left hand cell, this number is telling us, based on our sample data, that there is an $87.5 \%$ chance of measuring an aggregate less than $300 \mathrm{~nm}$ in size, given an $\mathrm{NaCl}$ concentration $<0.10 \mathrm{M}$ and given the presence of the NOM proxy tannic acid. Using a limited set of experimental data, these probabilities can be calculated in software programs designed for creating and updating BNs; for this project we are using the software package Hugin, which graphically illustrates the $\mathrm{BN}$ structure as well as the probability distributions for all variables. Based on uncertainty and data availability, choices will be made as to whether the conditional probabilities are calculated via the Maximum Likelihood Method (i.e. estimating the most likely distribution probability compatible with experiment) or the Bayesian Estimation method (trying to fit a $f(y \mid \theta)$ function. As more data become available, the prior values can be updated. For nodes such as $\mathrm{pH}$, which we know is important but for which we don't have data, there are multiple options. Laboratory experiments can be carried out. We can also elicit the conditional probabilities from experts by asking for a low $\mathrm{pH}$ and a given $\mathrm{NOM}$ and $\mathrm{NaCl}$ concentration state, which size bin would be expected. Although this example only considers one variable, aggregate size, as being described as a conditional probability (natural organic matter and salt concentration as treated as independent marginal probabilities), much more complex relationships can be represented using Bayesian networks with successive "generations" of variables that are interrelated by their conditional probabilities. 


\section{Feedback to improve nanomaterial design.}

The "green chemistry" prospects for nanomaterials are promising [38]. Products of nanochemistry and manipulation at the nanoscale will lead to the substitution of dangerous materials by nanomaterials and processes shown to pose less risk. Nanotechnology-inspired production will likely lead to more efficient use of materials and energy and an associated lower environmental footprint. However, the path forward with a green chemistry approach to reduce hazard is far from clear. The novel properties of nanomaterials that make them useful in a specific application are often the same properties that produce the hazard. For example just as a sharp knife makes it useful for cutting but may also injure, it is the capacity for reactive oxygen species (ROS ) generation by nanoscale $\mathrm{TiO} 2$ that makes it useful for degrading contaminants in water and causes concern over potential environmental impacts.

The possibility of designing nanomaterials to reduce hazard has been suggested as a means to ensure that nanostructured materials are "safe by design." However, it is impossible to anticipate every possible impact of a technology and therefore impossible to demonstrate that a technology is entirely "safe." At best, attempts to reduce hazard associated with a nanomaterial can only reduce risk and make them "safer by design." Moreover, success in "designing-out" hazard from non-nanomaterials (as opposed to designing materials to limit exposure) has been limited.

This approach implicitly assumes the ability to predict biological effects based on nanomaterial properties, a task that at best will yield long-term benefits but leave short-term demands for informed decision making in this field unsatisfied. The development of statistical relationships ranging from simple correlations to expert systems requires large data sets, produced, for example, using high throughput platforms. Even using such advanced discoverybased approaches, an already large space for exploration becomes even more vast when interactions with the wide range of environmental and physiological components that modify nanomaterials in actual systems are also taken into account. Also, the ability to predict to cases far outside the data set is likely to be limited at best. The de facto consequence of relying entirely on an uncertain future ability to understand the factors that control hazard as our first line of defense in managing risk, is to forego the goal of preventive action as the system develops while awaiting further information.

A more adaptive approach that is suited early-on to informing decisions in a precautionary fashion, while later moving to quantitative risk assessment, is one focused on managing risk through exposure. Due to the large number of possibilities for taking action early in the trajectory of a technology, managing exposure as the basis for risk management of an emerging technology such as nanotechnologies will likely prove to be more robust and successful. Exposure assessments based on quantities likely to be produced, project routes of 
exposure and persistence provide an immediate basis for identifying possible problems. Longterm, green chemistry approaches to designing nano-scale objects with limited environmental mobility, limited persistence, or limited bioavailability provide a wide range of options for mitigating risk by managing exposure. Mitigating exposure will be necessary to obtain the maximum benefits from nanotechnology, particularly those employing nanomaterials whose benefits are derived from the same properties that impart an inherent hazard. Important questions to be answered in evaluating nanomaterial risk are therefore related to the format that nanomaterials will be present in as commercial products, the potential for these materials to be released to the environment, and the transformations that those materials may undergo that affect their transport and potential for exposure.

\section{Concluding remarks: Implementation issues}

In a world of limited resources, one is tempted to choose between hazard assessment or exposure assessment as the basis for prioritizing research that will produce the required information to make informed early-stage forecasts of possible nanomaterials risks. However, both are clearly needed.

Similarly, one is tempted to trade-off short term needs for long-term advances in the fundamental science that will allow for truly predictive capability. Dilemmas such as these are partially resolved in cases where fundamental principles inform the development of simple, rapid measurements that can be applied to a broad number of nanomaterials (and their transformed variations) when these tests have relevance to both hazard and exposure. An example of such a measurement for conventional organic compounds is octanol water partition coefficient (Kow). The Kow informs transport and fate (e.g. effects on retarded transport in ground water) as well as biodistribution/ bioavailability for organic compounds (e.g. bioconcentration factor correlated with Kow). Analogous measurements such as nanoparticle affinity for reference surfaces, or macromolecule/ nanoparticles interactions might be developed along with standard protocols to inform elements of both hazard and exposure while building a foundation for long-term research that will allow for theory-based guidance on nanomaterial risks. 


\section{References Cited}

1. Hullmann, A., Who is winning the global nanorace? Nature Nanotechnology, 2006. 1: p. 81-83.

2 http://www.edf.org/documents/7287_Denison Testimony_10312007.pdf

3. Robichaud, C.O., et al., Estimates of upper bounds and trends in nano-TiO2 production as a basis for exposure assessment. Environmental Science \& Technology, 2009. 43(12): p. 4227-4233.

4. $\quad$ Murr, L.E., et al., Carbon nanotubes, nanocrystal forms, and complex nanoparticle aggregates in common fuel-gas combustion sources and the ambient air. J. Nano. Res., 2004. 6: p. 241-251.

5. $\quad$ Bang, J.J., et al., Carbon nanotubes and other fullerene nanocrystals in domestic propane and natural gas combustion streams. Journal of Nanoscience and Nanotechnology, 2004. 4(7): p. 716-718.

6. Heymann, D., et al., Fullerenes in the Cretaceous-Tertiary Boundary-Layer. Science, 1994. 265(5172): p. 645-647.

7. Becker, L., et al., Fullerenes in Meteorites and the Nature of Planetary Atmospheres, in Natural Fullerenes and Related Structures of Elemental Carbon, F.J.M. Rietmeijer, Editor. 2006, Springer, Netherlands. p. 95-121.

8. $\quad$ Hochella, M.F.J., et al., Nanominerals, Mineral Nanoparticles, and Earth Systems. Science, 2008. 319: p. 1631-1635.

9. $\quad$ Gutowski, T., et al., Thermodynamic Analysis of Resources Used in Manufacturing Processes. Environmental Science and Technology, 2009. 43(5): p. 1584-1590.

10. Robichaud, C.O., et al., Relative risk analysis of several manufactured nanomaterials: An insurance industry context. Envirionmental Science and Technology, 2005. 39(22): p. 8985-8994.

11. Plata, D.L., P.M. Gschwend, and C.M. Reddy, Industrially synthesized single-walled carbon nanotubes: compositional data for users, environmental risk assessments, and source apportionment. Nanotechnology, 2008. 19(18): p. 14.

12. Hansch, C. and T. Fujita, $\rho-\sigma-\pi$ Analysis. A method for the correlation of biological activity and chemical structure. J. Am. Chem. Soc., 1964. 86: p. 1616-1626.

13. Zhang, R. and A.J. Bivens, Comparing the use of bayesian networks and neural networks in response time modeling for service-oriented systems. Proceedings of the 2007 workshop on Service-oriented computing performance: aspects, issues, and approaches, 2007: p. 67-74.

14. Jensen, F.V. and T.D. Nielsen, Bayesian Networks and Decision Graphs. Second ed, ed. M. Jordan, J. Kleinberg, and B. Schölkopf. 2007, New York, NY: Springer Science + Business Media, LLC.

15. Auffan, M., et al., Enhanced adsorption of arsenic onto nano-maghemites: As(III) as a probe of the surface structure and heterogeneity. Langmuir, 2008. 24: p. 3215-3222.

16. Auffan, M., et al., Nanomaterials as adsorbant, in Environmental nanotechnology - applications and impacts of nano-materials, ed. M.R. Wiesner and J.Y. Bottero. 2007, New York: MacGrawHill.

17. Bailey, D.A., et al., Characterization of alumoxane-derived ceramic membranes. J. Membrane Sci., 2000. 176: p. 1-9.

18. Brant, J., et al., Comparison of Electrokinetic Properties of Colloidal Fullerenes (n-C60) Formed Using Two Procedures. Environ. Sci. Technol., 2005. 39(17): p. 6343-6351.

19. Brant, J.A., et al., Nanoparticle transport, aggregation, and deposition, in Environmental Nanotechnology, M.R. Wiesner and J.Y. Bottero, Editors. 2007, McGraw-Hill: New York, NY.

20. Brant, J.A., et al., Fullerol cluster formation in aqueous solutions: Implications for environmental release. Journal of Colloid and Interface Science, 2007.

21. Brant, J.A., et al., Characterizing the impact of preparation method on fullerene cluster structure and chemistry. Langmuir, 2006. 22: p. 3878-3885.

22. Brant, J.A., et al., Comparison of electrokinetic properties of colloidal fullerenes (n-C60) formed using two procedures. Environmental Science \& Technology, 2005. in press.

23. Brant, J.A., H. Lecoanet, and M.R. Wiesner, Aggregation and Deposition Characteristics of Fullerene Nanoparticles in Aqueous Systems. Journal of Nanoparticle Research, 2005. 7: p. 545-553. 
24. Callender, R.L., et al., Aqueous synthesis of water soluble alumoxanes: environmentally benign precursors to alumina and aluminum-based ceramics. Chemistry of Materials, 1997. 9: p. 2418-2433.

25. Cortalezzi, M.M., et al., Characteristics of ceramic membranes derived from alumoxane nanoparticles. Journal of Membrane Science, 2002. 205: p. 33-43.

26. Cortalezzi, M.M., et al., Ceramic membranes derived from ferroxane nanopartices: a new route for the fabrication of iron oxide ultrafiltration membranes. J. Membrane Sci., 2003. 227(207-217).

27. Cortalezzi, M.M., C. V., and M.R. Wiesner, Controlling nanoparticle template morphology: effect of solvent chemistry. Colloid and Interface Science, 2005. 283: p. 366-372.

28. Hoffman, M., E.M. Hotze, and M.R. Wiesner, Reactive Oxygen generation on nanoparticulate material, in Environmental Nanotechnology, M.R. Wiesner and J.Y. Bottero, Editors. 2007, McGraw-Hill: New York, NY.

29. Hotze, E.M., et al., Mechanisms of photochemistry and reactive oxygen production by fullerene suspensions in water. Environmental Science and Technology, 2008. 42(11): p. 4175-4180.

30. Jolivet, J.P. and A.R. Barron, Nanomaterials fabrication, in Environmental nanotechnology - applications and impacts of nano-materials, ed. M.R. Wiesner and J.Y. Bottero. 2007, New York: MacGrawHill.

31. Lecoanet, H.F., J.-Y. Bottero, and M.R. Wiesner, Laboratory assessment of the mobility of nanomaterials in porous media. Environmental Science \& Technology, 2004. 38: p. 5164-5169.

32. Lecoanet, H.F. and M.R. Wiesner, Velocity effects on fullerene and oxide nanoparticle deposition in porous media. Environmental Science \& Technology, 2004. 38(16): p. 4377-4382.

33. Pickering, K.D. and M.R. Wiesner, Fullerol-sensitized production of reactive oxygen species in aqueous solution. Environmental Science \& Technology, 2005. 39(5): p. 1359-1365.

34. Rose, J., et al., Synthesis and characterization of carboxylate-FeOOH nanoparticles (ferroxanes) and ferroxane-derived ceramics. Chemistry of Materials, 2002. 14(2): p. 621-628.

35. Xia, T., et al., Comparison of the abilities of ambient and manufactured nanoparticles to induce cellular toxicity according to an oxidative stress paradigm. Nano Lett., 2006. 6(8): p. 1794-1807.

36. Derjaguin, B.V. and L.D. Landau, Theory of the stability of strongly charged lyophobic sols and of the adhesion of strongly charged particles in solutions of electrolytes. Acta Physicochim. URSS, 1941. 14: p. 733-762.

37. Verwey, E.J.W. and J.T.G. Overbeek, Theory of the Stability of Lyophobic Colloids. 1948, Amsterdam: Elsevier.

38 Lancaster, M, Green Chemistry.An Introductary Text. Royal Society of Chemistry. 2010, Cambridge 